\title{
Síndrome metabólico en individuos de una comunidad rural
}

\author{
Metabolic syndrome in individuals in a rural \\ community
}

\author{
Andreína Mari, Yanetxy Marval, Ana Karina Suárez, Everildda Arteaga, \\ Elisa Martínez, Gilberto Bastidas • Carabobo (Venezuela)
}

\section{Resumen}

Objetivo: caracterizar el síndrome metabólico (SM) en un medio rural venezolano.

Contexto y tipo de estudio: la frecuencia de SM es elevada en el mundo y es elemento de riesgo en el desarrollo de diabetes mellitus y enfermedad cardiovascular, pero existe limitada información sobre SM en las zonas rurales de la mayoría de los países. Se realizo un estudio descriptivo y transversal en un sector rural del estado Cojedes, Venezuela.

Material y método: fueron 39 los sujetos con edades entre 20-59 años, 51.3\% mujeres y 56.4\% tenían antecedentes familiares de riesgo cardiometabólico. Se definió según el Tercer Reporte del Panel de Expertos del Programa Nacional de Educación (NCEP/ATPIII), como SM a la presencia de por lo menos tres de los cinco factores siguientes: obesidad abdominal ( $>88 \mathrm{~cm}$ en mujeres y de $102 \mathrm{~cm}$ en hombres) hiperglicemia en ayunas (>110 mg/dL), hipertensión arterial (>130/85 mmHg), lipoproteína de alta densidad (HDL-c) $(<50 \mathrm{mg} / \mathrm{dL})$ y triglicéridos elevados $(>150 \mathrm{mg} / \mathrm{dL})$.

Resultados: la prevalencia de SM fue $20.5 \%, 12.8 \%$ hombres y $7.7 \%$ mujeres, el criterio diagnóstico predominante fue la hipertrigliceridemia (53.8\%) y las mujeres con SM eran más hipertensas, hiperglicémicas, con menos HDL-c, mayor concentración de triglicéridos y obesas que los hombres.

Conclusión: el SM afecta por igual al medio rural y urbano, pero en Venezuela la prevalencia es mayor en la región urbana llanera. El género masculino es también el más afectado y la diabetes mellitus e HTA son los antecedentes familiares más frecuentes. La obesidad abdominal e hipertrigliceridemia los factores de riesgo SM más señalados. Se infiere adopción rural de hábitos alimentarios urbanos. (Acta Med Colomb 2012; 37: 177-182)

Palabras clave: síndrome metabólico, diabetes tipo 2, enfermedad cardiovascular, factores de riesgo, área rural.

\section{Abstract}

Objective: to characterize the metabolic syndrome (MS) in a rural Venezuelan environment.

Context and Type of Study: MS has a high frequency worldwide and is a risk factor in the development of diabetes mellitus and cardiovascular disease, but the information about SM in these areas is limited. We performed a cross-sectional descriptive study in a rural sector in Cojedes state, Venezuela.

Methods: we studied 39 subjects aged 20-59 years. 51.3\% female and 56.4\% had a family history of cardiometabolic risk. According to the Third Report of the Expert Panel of the National Education Program (NCEP / ATPIII), SM was defined as the presence of at least three of the following five factors: abdominal obesity ( $>88 \mathrm{~cm}$ in women and $102 \mathrm{~cm}$ in men) fasting hyperglycemia (> $110 \mathrm{mg} / \mathrm{dL}$ ), hypertension (> 130/85 mmHg), high density lipoprotein (HDL-C) $(<50 \mathrm{mg} / \mathrm{dL}$ ) and triglycerides (> $150 \mathrm{mg} / \mathrm{dL}$ )

Results: the prevalence of MS was $20.5 \%$ (12.8\% men and $7.7 \%$ women). The predominant diagnostic criteria was hypertriglyceridemia (53.8\%) and women with MS were more hypertensive, hyperglycemic, with less HDL-C, higher triglycerides, and were more obese than men.

Conclusion: MS equally affects both rural and urban areas, but in Venezuela the prevalence is higher in urban plains. Male gender is also the most affected and diabetes mellitus and hypertension are the most frequent family history. Abdominal obesity and hypertriglyceridemia are the most signaled SM risk factors. It infers the rural adoption of urban food habits. (Acta Med Colomb 2012; 37: 177-182)

Keywords: metabolic syndrome, type 2 diabetes, cardiovascular disease, risk factors, rural area.

Dres. Andreína Mari, Yanetxy Marval, Ana Karina Suárez: Médicos Cirujanos. Facultad de Ciencias de la Salud, Universidad de Carabobo; Dr. Everilda Arteaga: Médico Especialista en Pediatría y Adolescencia, Departamento Clínico Integral del Norte, Escuela de Medicina, Universidad de Carabobo; Dra. Elisa Martínez: Licenciada en Bioanálisis. Departamento de Investigación y Desarrollo Profesional, Escuela de Bioanálisis, Universidad de Carabobo; Dr. Gilberto Bastidas: Médico Cirujano, MSc en Gerencia de la Educación, MSc en Protozoología y Dr. en Parasitología. Departamento de Salud Pública, Escuela de Salud Pública y Desarrollo Social, Facultad de Ciencias de la Salud, Universidad de Carabobo. Carabobo (Venezuela).

Correspondencia. Dr. Gilberto Bastidas, Carabobo (Venezuela).

E-mail: bastidasprotozoo@hotmail.com Recibido: 30/I/2012 Aceptado: 25/X/2012 


\section{Introducción}

El síndrome metabólico (SM) es definido como una constelación de alteraciones en la fisiología humana que se constituyen como elementos de riesgos en el desarrollo de diabetes mellitus tipo 2 y en enfermedad cardiovascular, enfermedades de gran impacto social y sanitario por su alta prevalencia, complicaciones crónicas y alta mortalidad relacionada. El SM incluye al menos tres de los siguientes factores: la obesidad abdominal (circunferencia de cintura $>102 \mathrm{~cm}$ en hombres y $>88 \mathrm{~cm}$ en mujeres), hipertensión (presión sanguínea $\geq 135 / 80 \mathrm{mmHg}$ ), hiperglicemia y/o intolerancia a la glucosa (glicemia $\geq 100 \mathrm{mg} / \mathrm{dL}$ ), dislipidemia (caracterizada por los bajos niveles de lipoproteínas de alta densidad [HDL-C; $<40 \mathrm{mg} / \mathrm{dL}$ en mujeres y $<50$ $\mathrm{mg} / \mathrm{dL}$ en hombres] y altos niveles de triglicéridos $[\geq 150$ $\mathrm{mg} / \mathrm{dL}])(1-4)$.

La prevalencia de SM es elevada en el mundo, con rangos entre $10-84 \%$, en dependencia con la edad, región, medio ambiente, etnicidad y la definición de SM empleada. Por tanto, es considerado un importante problema de salud pública global asociado con incremento de hasta cinco veces en la prevalencia de diabetes tipo 2 y entre dos y tres veces en la frecuencia de aparición de las enfermedades cardiovasculares, enfermedades que podrían agotar el presupuesto destinado al sector sanitario de países desarrollados o en vías de lograrlo, por ubicarse entre las primeras causas de morbimortalidad en el mundo (2, 5-8).

Ahora bien, en Europa la prevalencia de SM supera el $20 \%$ en los hombres, pero es de $12 \%$ en las mujeres y el grupo de edad mayormente afectado se ubica entre 40-55 años (9). En Asia la prevalencia es relativamente más baja aunque variable de acuerdo con las diferentes etnias (12-20\%) (10). En América del Norte, específicamente en Estados Unidos la prevalencia de SM es de $24 \%$, se comporta igual en hombres y mujeres pero incrementa su frecuencia con la edad con valores de $6.7 \%$ en personas entre 20-29 años y hasta de $43.5 \%$ en las edades comprendidas entre 60 y 69 años (11). En América Latina la prevalencia de SM oscila entre 22-33\%, según muestran estudios realizados en Argentina, Brasil, Chile, Colombia y México, con similar afectación por grupos de edad a la observada en Estados Unidos (12-17).

En Venezuela la prevalencia también se ubica dentro de estos rangos según estudios realizados en los estados Lara y Zulia y también compromete los grupos de edad considerados internacionalmente como económicamente productivos, población, este grupo de edad, que por el estresante estilo de vida, pues están en constante actividad, no vigila sus hábitos alimentarios e ingieren mayor cantidad de grasas (saturadas y grasas trans) y carbohidratos (azúcares refinados) que de fibras (12-17).

La adaptación de las comunidades rurales a los estilos de vida moderna de las grandes ciudades ha hecho mayor la afluencia de sujetos por enfermedad cardiovascular crónica a la consulta ambulatoria rural, muchos con claros signos de hipertensión y obesidad abdominal, factores de riesgo que pueden potenciar la aparición de esta patología cardiaca y también de diabetes mellitus tipo 2, por la sustitución de los hábitos alimentarios propios del campo (rica en productos del campo, naturales, con mucha fibra y constante ejercicio), por la que identifican a las grandes ciudades (ingesta de escasa fibra, sedentarias y en estrés constante) (9).

Además, existe limitada información sobre prevalencia de SM en Venezuela, especialmente en las zonas agrícolas y ganaderas, motivos estos que justificaron el presente estudio sobre el comportamiento del SM en una población rural, específicamente en Cojedes, uno de los estados llaneros de Venezuela, con el objeto de también identificar factores de riesgo de enfermedades cardiacas y metabólicas crónicas, información que permitirá abortar complicaciones si con la misma se desarrollan programas de salud que modifiquen conductas perjudiciales, que reflejan estilos de vida poco saludables en relación directa con fenómenos de transición económica, urbanización, globalización e industrialización.

\section{Contexto y tipo de estudio \\ Material y métodos}

Se trató de un estudio descriptivo, transversal y de campo realizado a finales del año 2011 en el sector El Castaño, una comunidad rural del llano venezolano con escasa, pero no ausente, transculturización y flujo migratorio, comunidad que pertenece al municipio Lima Blanco, estado Cojedes, Venezuela; zona conformada por 38 casas, de las cuales 22 se encuentran habitadas (información obtenida mediante visita al área y conteo de los hogares), en promedio el grupo familiar consta de cuatro personas entre adultos y niños. En total fueron 39 las personas adultas con edades comprendidas entre 20-59 años las que participaron en el estudio (el total de la población adulta de la comunidad estudiada), sin criterios de exclusión solo el deseo de participar en el estudio, previa explicación de los objetivos de la investigación y firma del consentimiento informado según lo establecido por las normas de investigación clínica.

Para estipular la incidencia de SM se recurrió a un instrumento para la recolección de información con tres secciones. La primera incluía los datos generales de identificación y filiación, la segunda recogía datos sobre: hábitos psicobiológicos (consumo de café, alcohol y fumar), antecedentes personales y familiares patológicos en primer grado de consanguinidad (19). La tercera y última sección incluyó la medición de aspectos cuya alteración determinan la presencia de SM, como la circunferencia de cintura (con cinta métrica flexible e inextensible colocada a la altura del punto medio entre la ultima costilla y la cresta iliaca, con el sujeto en bipedestación al final de la espiración no forzada) (20), presión arterial (con esfigmomanómetro de mercurio, por el método auscultatorio, en posición sentada, posterior a 15 minutos de reposo del sujeto, y la medición fue realizada en dos ocasiones por el mismo observador) (21), glicemia, lipoproteínas de alta densidad y triglicéridos. Del mismo modo, se midió el peso y talla de cada individuo sin zapatos 
y con vestimenta ligera mediante balanza con estadiómetro previamente calibrada.

Para las determinaciones de parámetros sanguíneos se extrajeron luego de 12 horas de ayuno $10 \mathrm{~mL}$ de sangre por venopunción de vaso sanguíneo periférico braquial con jeringa estéril. Las muestras fueron vertidas en tubos de ensayo sin anticoagulante y centrifugadas para la obtención del suero. En la cuantificación de las concentraciones de glucosa, colesterol, triglicéridos y de lipoproteínas se emplearon los estuches de la casa comercial Wiener Lab®, basado en un método enzimático-colorimétrico. La lectura espectrofotométrica se hizo automáticamente en un equipo conocido como Express Plus 550®.

Se definió, según el Tercer Reporte del Panel de Expertos del Programa Nacional de Educación (NCEP/ATPIII siglas en inglés) (2) como SM a la presencia de por lo menos tres de los cinco factores siguientes: obesidad abdominal (> 88 $\mathrm{cm}$ en mujeres y $>102 \mathrm{~cm}$ en hombres) hiperglicemia en ayunas $(>110 \mathrm{mg} / \mathrm{dL})$, hipertensión arterial $(>130 / 85 \mathrm{~mm}$ $\mathrm{Hg}$, ) lipoproteína de alta densidad (HDL-c) $(<50 \mathrm{mg} / \mathrm{dL})$ y triglicéridos elevados ( $>150 \mathrm{mg} / \mathrm{dL})$. El índice de masa corporal (IMC) se calculó mediante la ecuación: peso (kg)/ $(\text { talla })^{2}(\mathrm{~m})$ y en la evaluación nutricional se honraron los valores de índice de masa corporal (IMC) establecidos por la OMS (22), así se consideran en déficit nutricional a aquellos sujetos con IMC menor a $18.5 \mathrm{~kg} / \mathrm{m}^{2}$, en normopeso a individuos con un IMC $\geq 18.5$ pero que no supere los $24.9 \mathrm{~kg} / \mathrm{m}^{2}$, en sobrepeso cuando el intervalo de IMC se encuentra entre $25-29.9 \mathrm{~kg} / \mathrm{m}^{2}$ y finalmente en obesidad cuando es igual o superior a $30 \mathrm{~kg} / \mathrm{m}^{2}$.

\section{Análisis estadístico}

Los datos se registraron y analizaron mediante el programa estadístico SPSS/PC (versión 11.0 para Windows, SPSS, Chicago, IL). Para esta investigación se aceptó un error estándar de 5\% y nivel de confianza de $95 \%$. Además de estadísticas descriptivas se utilizó en la comparación de parámetros (medias) la prueba t de Student, y la prueba de Chi cuadrado $\left(x^{2}\right)$ para determinar asociación entre variables cualitativas y de proporciones.

\section{Resultados}

De los 39 sujetos estudiados 51.3\% (20/39) pertenecían al género femenino y $48.7 \%$ (19/39) al masculino, obviamente sin diferencia significativa entre género $(\mathrm{p}=0.82)$, debida esta circunstancia a que los adultos conviven mayoritariamente como parejas. Las personas entre 30-39 años representan $64.1 \%$ de la muestra y el resto de la proporción se distribuye entre los grupos de edad comprendidos entre: 40-49 años (15.4\%), 20-29 años (12.8\%) y $\geq 50$ años (7.7\%) (con diferencia significativa entre estos tres grupos de edades y las personas entre 30-39 años de edad [p $<0.01]$ ).

De los sujetos estudiados $56.4 \%$ tenían antecedentes familiares de enfermedad cardiometabólica, de este porcentaje correspondió a la diabetes mellitus algo más de la mitad $(56.4 \%)$ y a HTA $43.6 \%$. Igualmente, la prevalencia de factores de riesgo asociado al SM en sujetos de la muestra fue de $90 \%$ para el cafeínico, $48 \%$ para la ingesta de alcohol y sólo $10 \%$ de los individuos encuestados tiene hábitos tabáquicos (fuman al menos 30 cigarrillos diarios y desde hace cinco años en promedio).

Fueron cinco los criterios diagnósticos de SM hallados, de éstos el más frecuentemente alterado fue: los triglicéridos elevados, pues estuvo presente en 21 de los 39 sujetos que participaron en el estudio (Tabla 1). La prevalencia de SM fue de $20.5 \%$, es decir, siete de 39 sujetos exhibían al menos tres de los criterios establecidos para definir el padecimiento de SM y uno de los individuos tenía cuatro de los mismos (Tabla 2 y 3). Las mujeres con SM eran más hipertensas, hiperglicémicas, con menos niveles de HDL-c, mayor concentración sérica de triglicéridos y obesidad abdominal que los hombres ( $\mathrm{p}<0.05$ para todos).

La valoración del estado nutricional antropométrico reveló que las mujeres con SM tenían en su mayoría obesidad $(33.3 \%)$ o sufrían de sobrepeso $(66.7 \%)$, sin diferencias significativas entre estas y los hombres $(\mathrm{p}=0.5)$ (Tabla 4).

Tabla 1. Comparación de prevalencias de factores de riesgo de síndrome metabólico en sujetos del sector rural El Castaño, municipio Lima Blanco, estado Cojedes, Venezuela.

\begin{tabular}{|c|c|c|c|c|}
\hline \multirow{3}{*}{ Factores de riesgo } & \multicolumn{4}{|c|}{ Sujetos* } \\
\hline & \multicolumn{2}{|c|}{$\begin{array}{c}\text { Mujeres** } \\
(\mathbf{n}=\mathbf{2 0})\end{array}$} & \multicolumn{2}{|c|}{$\begin{array}{l}\text { Hombres } \\
(\mathbf{n}=19)\end{array}$} \\
\hline & f & $\%$ & f & $\%$ \\
\hline HDL-c $(<50 \mathrm{mg} / \mathrm{dL})$ & 7 & 35 & 5 & 26,3 \\
\hline Hiperglicemia (>110mg/dL) & 8 & 40 & 9 & 47,4 \\
\hline Hipertensión (>130/85mm Hg) & 7 & 35 & 5 & 26,3 \\
\hline Triglicéridos elevados (>150 mg/dL) & 12 & 60 & 8 & 42,1 \\
\hline $\begin{array}{l}\text { Obesidad abdominal ( }>88 \mathrm{~cm} \text { en mujeres } \\
\mathrm{y}>102 \mathrm{~cm} \text { en hombres) }\end{array}$ & 19 & 95 & 14 & 73,7 \\
\hline $\begin{array}{l}* \text { Prevalencia calculada respecto a número } \\
* * \mathrm{p}<0.05\end{array}$ & . & & & \\
\hline
\end{tabular}

Tabla 2. Sujetos agrupados según el número de criterios diagnósticos de síndrome metabólico del sector rural El Castaño, municipio Lima Blanco, estado Cojedes, Venezuela.

\begin{tabular}{|c|cc|}
\hline \multirow{2}{*}{ Número de criterios diagnósticos } & \multicolumn{2}{|c|}{ Sujetos } \\
\cline { 2 - 3 } & $\mathbf{f}$ & $\%$ \\
\hline $\mathbf{1}$ & 10 & 25.6 \\
$\mathbf{2}$ & 12 & 30.8 \\
$\mathbf{3}$ & 9 & 23.1 \\
$\mathbf{4}$ & 7 & 17.9 \\
$\mathbf{5}$ & 1 & 2,6 \\
\hline Total & 0 & 0 \\
\hline
\end{tabular}


Tabla 3. Prevalencias de síndrome metabólico en sujetos del sector rural El Castaño, municipio Lima Blanco, estado Cojedes, Venezuela.

\begin{tabular}{|c|c|c|}
\hline \multirow[t]{2}{*}{ Género** } & \multicolumn{2}{|c|}{ Sujetos con SM* } \\
\hline & f & $\%$ \\
\hline Femenino & 3 & 7,7 \\
\hline Masculino & 5 & 12,8 \\
\hline Total & 8 & 20,5 \\
\hline $\begin{array}{l}\text { *Prevalenci } \\
* * \mathrm{p}=0.31\end{array}$ & & \\
\hline
\end{tabular}

Tabla 4. Comparación entre el estado nutricional antropométrico entre mujeres y hombres con y sin síndrome metabólico. Sector rural El Castaño, municipio Lima Blanco, estado Cojedes, Venezuela.

\begin{tabular}{|c|c|c|c|c|}
\hline \multirow{3}{*}{ Evaluación nutricional } & \multicolumn{4}{|c|}{ Sujetos con SM* } \\
\hline & \multicolumn{2}{|c|}{$\begin{array}{c}\text { Mujeres } \\
(n=3)\end{array}$} & \multicolumn{2}{|c|}{$\begin{array}{c}\text { Hombres } \\
\quad(n=5)\end{array}$} \\
\hline & $\mathbf{f}$ & $\%$ & $\mathbf{f}$ & $\%$ \\
\hline Déficit & 0 & 0 & 0 & 0 \\
\hline Normal & 0 & 0 & 1 & 20 \\
\hline Sobrepeso** & 1 & 33,3 & 1 & 20 \\
\hline Obesidad ** & 2 & 66,7 & 3 & 60 \\
\hline
\end{tabular}

\section{Discusión}

La prevalencia de SM hallada fue de $20.5 \%$, duplica esta proporción a la encontrada en otro sector rural venezolano (único abordado hasta ahora y que tiene una densidad poblacional similar a la población objeto de este estudio), específicamente del estado Mérida, una entidad federal de la región andina venezolana (9\%), con diferente geografía y costumbres, respecto a la región del llano venezolano en este estudio investigada, por tanto, los resultados reportados muestran la existencia de distinta prevalencia de SM en el medio rural venezolano, esto es particularmente interesante porque la muestra representó $78 \%$ de la población examinada (23).

La diferencia entre género respecto a la prevalencia de SM en este medio rural, aunque sin diferencias significativas estuvo a favor del masculino, que también es lo esperado, salvando la diferencia poblacional, para muchas regiones urbanas del mundo (únicas con las cuales realizar comparación debido a la pobre información que se posee de este fenómeno en el medio rural) (24-25), es así, que en el país, pero para una población urbana adolescente, se muestran prevalencias de hasta $6.2 \%$ en varones referente a $2.1 \%$ de las mujeres (15). Así mismo destaca en el medio rural la importante prevalencia que mostraron cada uno de los factores de riesgo que caracterizan al SM, todas por encima, a pesar de la menor población rural revisada, de las reportadas en el estado Zulia (Venezuela) e incluso en algunas regiones de Chile y Colombia (13, 26-28).
La diabetes mellitus y la HTA son los antecedentes familiares mayormente reportados en los sujetos estudiados, pero también se detectaron los hábitos cafeícos, el consumo de alcohol y tabaco. Queda claro que la presencia de éstos y otros factores influyen en la aparición de SM y a la larga en el desarrollo de enfermedades circulatorias, pues se ubican dentro de los más de 250 factores asociados con el desarrollo de estas patologías, factores estos entre los cuales también se incluyen: la hipercolesterolemia, edad, historia familiar, inflamación, adhesión celular, estrés oxidativo y disfunción endotelial, entre otros $(29,30)$.

Llama poderosamente la atención que más de $20 \%$ de los sujetos con SM tienen hábitos tabáquicos, sustancia que una vez inhalada interfiere con la síntesis de piridoxal fosfato lo que se traduce en aumento de la homocisteína plasmática, que estimula la formación de ateromas en acción sinérgica con otros factores de riesgo $(31,32)$.

En primer lugar la obesidad abdominal (asociada con estado proinflamatorio y acumulación de grasa ectópica en hígado, corazón, músculo y páncreas) un factor de riesgo independiente para el desarrollo de enfermedad coronaria (33-35) y en segundo lugar la hipertrigliceridemia, fueron los factores de riesgo predominantemente encontrados en los sujetos estudiados. Factores principalmente la hipertrigliceridemia (debido al aumento del triacilgliceridos a partir de las VLDL), que incrementan el riesgo de depósitos grasos y por tanto de lesiones en las arterias coronarias y aorta por el desorden inflamatorio crónico que se produce en la íntima arterial durante la formación del ateroma (36-43). Ahora bien, los dos factores de riesgo de SM en este estudio señalados como preponderantes, también lo fueron para la población zuliana y para poblaciones andinas venezolanas según estudios realizados por Flórez (13) y Becerra (44), entonces podría presumirse por la contundencia de lo hallado, guardando la diferencia poblacional entre comunidades, que estos factores sean los más altos contribuyentes de la prevalencia de SM en la población venezolana, se trate de rural o urbana.

Los datos muestran que más de $60 \%$ de hombres y mujeres con SM tienen obesidad como anormalidad en el peso corporal, lo mismo fue reportado, pero en mujeres de comunidades urbanas (en consecuencia con mayor población) de Estados Unidos y localmente (en Venezuela) en Mucuchiés una región urbana de Mérida, esto es particularmente importante, porque en estos sujetos existen otros factores de riesgo cardiometabólico, individuos que pueden presentar tres o más componentes del SM y en frecuencia superior que las personas con sobrepeso, como sucedió con los sujetos obesos de esta investigación (45-47). Así mismo fue significativamente mayor la circunferencia abdominal en las mujeres, a esta diferencia se agrega como en el estudio de Mucuchiés un comportamiento dislipidémico diferencial marcado por mayor hipertrigliceridemia y frecuencia de bajos niveles de HDL-c en mujeres $(44,48)$.

Se menciona en tercer lugar a la hiperglicemia como factor de riesgo, elemento que produce daño en diferentes 
órganos del cuerpo humano, sin embargo, en este parámetro por ser susceptible a alteración por diversos factores, una sola medición de incremento no necesariamente indica patología subyacente, se requiere entonces de estudios complementarios que confirmen la presencia de verdadera lesión $(15,49)$. La hiperglicemia aunque está presente en más del $40 \%$ de los sujetos con SM es significativamente mayor en hombres y la frecuencia, alta, de HTA descubierta en ambos géneros fue de $34 \%$.

Lo hallado en este estudio contraría la creencia popular venezolana que en el campo se vive y come mejor, pues en la génesis de SM desempeña importante papel la alimentación, en este caso la inadecuada, puede inferirse entonces que nuestros campesinos, quizás por transculturización, han adoptado los hábitos alimentarios de las pobladores de las ciudades, el urbanismo puede ya estar en los campos o que simplemente lo que comen no es lo adecuado y el SM ya estaba allí, sólo que no había sido encontrado.

Pero se hace necesario estudiar otras regiones rurales venezolanas para determinar la verdadera dimensión del SM en estas regiones y comprobar si se trata de un problema de salud pública, máxime que por las revelaciones de intervenciones hechas en otras regiones del mundo, se sabe del adecuado conocimiento sobre diabetes, percepción de riesgo y expectativas de éxito que poseen los grupos sociales respecto al impacto de la enfermedad cardiovascular (50). Lo crucial como recomendación es el seguimiento médico de los individuos afectados con SM, para evitar complicaciones mediante la promoción, educación y fomento de la salud, con el fin de que adquieran conductas saludables que impliquen la modificación de hábitos psicobiológicos y alimentarios.

\section{Declaración de conflicto de interés}

Los autores declaran no tener conflicto de interés.

\section{Referencias}

1. Wilson P, D'Agostino R, Levy D, Belanger A, Silbershatz H, Kannel W Prediction of coronary heart disease using risk factors categories. Circulation 1990; 97: 1837-47.

2. Third report of the national colesterol education program. Expert panel on detection, evaluation, and treatment of high blood cholesterol in adults (Adult treatment panel III) final report. Circulation 2002; 106: 3143-21.

3. León I. Guías para el diagnóstico y tratamiento del síndrome metabólico y la diabetes tipo 2 (DM2). Nuevos criterios. Salus 2004; 8: 1-3.

4. Grundy $\mathbf{S}$. Criterios actuales para el diagnóstico y tratamiento del síndrome metabólico. Rev Endrocrinol Metabol 2005; 112:2735-52.

5. Sandhofer A, Kaser S, Ritsch A, Laimer M, Engl J, Paulweber B, et al. Cholesteryl ester transfer protein in metabolic syndrome. Obesity 2006; 14: 812-8.

6. Desroches $\mathbf{S}$, Lamarche $\mathbf{B}$. The evolving definitions and increasing prevalence of the metabolic syndrome. Appl Physiol Nutr Metab 2007; 32: 23-32.

7. Ministerio de Salud de Venezuela. Anuario de Estadística Vital [Internet] 2006 [citado el 4 de marzo de 2011]. Disponible en:htpp://www.mpps.gob.ve/ms/ direcciones_msds/Epidemiologia/estadística/Archivo/Anuarios.htm.

8. Kolovou GD, Anagnostopoulou KK, Salpea KD, Mikhailidis DP. The prevalence of metabolic syndrome in various populations. Am J Med Sci 2007; 333: 362-71.

9. Sánchez A. El síndrome metabólico. Rev Soc Esp Med Interna 2005; 2: 3-4.

10. The DECODA Study Group. Prevalence of the metabolic syndrome in populations of Asian origin. Comparison of the IDF definition with the NCEP definition. Diab Res Clin Pract 2007; 76: 5767-71.

11. Ford ES, Giles WH, Mokdad AH. Increasing prevalence of the metabolic syndrome among U.S. adults. Diabetes Care 2004; 27: 2444-49.

12. Rodgers A, Ezzati M, Vander Hoorn S, Lopez AD, Lin RB, Murray CJ. Comparative Risk Assessment Collaborating Group. Distribution of major health risks: findings from the Global Burden of Disease study. PLoS Med 2004; 1: e27. 13. Florez H, Silva E, Fernandez V, Ryder E, Sulbaran T, Campos G, et al. Prevalence and risk factors associated with the metabolic syndrome and dyslipidemia in White, Black, Amerindian and Mixed Hispanics in Zulia State, Venezuela. Diabetes Res Clin Pract 2005; 69: 63-7.

14. Barria RM, Amigo H. Transición Nutricional. Una revisión del perfil Latinoamericano. ALAN 2006; 56: 3-11.

15. Lozada M, Machado S, Manrique M, Martínez D, Suárez O, Guevara H. Factores de riesgo asociados al síndrome metabólico en adolescentes. Gac Méd Caracas 2008; 116: 323-9.

16. Escobedo J, Schargrodsky H, Champagne B, Silva H, Boissonnet CP, Vinueza R, et al. Prevalence of the Metabolic Syndrome in Latin America and its association with sub-clinical carotid atherosclerosis: the CARMELA cross sectional study. Cardiovasc Diabetol 2009; 8: 1-9.

17. Hudson JI, Lalonde JK, Coit CE, Tsuang MT, McElroy SL, Crow SJ, et al. Longitudinal study of the diagnosis of components of the metabolic syndrome in individuals with binge-eating disorder. Am J Clin Nutr 2010; 91: 1568-73.

18. World Medical Association declaration of Helsinki. Recommendations guiding physicians in biomedical research involving human subjects. JAMA 1997; 27711: $925-6$.

19. Rodríguez-Larralde A, Mijares ME, Nagy E, Espinosa R, Ryder E, DiezEwald MP et al. Relación entre el nivel socioeconómico y hábitos de vida, con el fibrinógeno y el factor von willebrand en venezolanos sanos y con cardiopatía isquémica. Invest Clin 2005; 46: 157-68.

20. Lohman TG, Roche AF, Martorell R. Anthropometric standardization reference manual. Champaign, IL: Human Kinetics Books 1988.

21. Joint National Committee on Prevention, Detection, Evaluation, and Treatment of High Blood Pressure. The seventh report of the joint national committee on prevention, detection, evaluation, and treatment of high blood pressure. JAMA 2003; 289: 2560-71.

22. WHO. Physical status: the use and interpretation of anthropometry. Report of a WHO Expert Committee. World Health Organ Tech Rep Ser 1995; 854: 1-452.

23. Molina-Lobo M, Velázquez-Maldonado E. Prevalencia del síndrome metabólico en dos poblaciones del Estado Mérida. Comparación según definición del NCEP/ ATP-III e IDF. Resumen. XVII Congreso Panamericano de Endocrinología y XI Congreso Venezolano de Endocrinología y Metabolismo, Margarita,Venezuela 2008. Rev Venez Endocrinol Metab 2009; 7: 39

24. Medina-Lezama J, Zea-Díaz H, Morey-Vargas O, Bolaños-Salazar J, Muñoz-Atahualpa E, Postigo-MacDowall M, et al. Prevalence of the metabolic syndrome in Peruvian Andean Hispanics. The Prevention study. Diabetes Res Clin Pract 2007; 78: 270-81.

25. Kawada T, Inagaki H, Wakayama Y, Li Q, Katsumata M. Serum insulin is significantly related to components of the metabolic syndrome in Japanese working men. Clin Hypertens (Greenwich) 2010; 12: 309-14.

26. Bautista L, Oróstegui M, Vera L, Prada G, Orozco L, Herrán O. Prevalence and impact of cardiovascular risk factors in Bucaramanga, Colombia: results from the Countrywide Integrated Noncommunicable Disease Intervention Programme (CINDI/ CARMEN) baseline survey. Eur J Cardiovasc Prev Rehabil 2006; 13: 769-75

27. Palomo I, Icaza G, Mujica V, Núñez L, Leiva E, Vásquez M, et al. Prevalencia de factores de riesgo cardiovascular clásicos en población adulta de Talca, Chile, 2005. Rev Med Chile 2007; 135: 904-12.

28. Bermúdez V, Cano C, Arraiz N, Amell A, Cabrera M, Reyna N, et al. The Maracaibo City Metabolic Syndrome Prevalence Study: Design and Scope. Am J Ther 2010; 17: 288-94.

29. Choy P, Mynin D, Zhu Q, Dakshinamurti K, Karmin O. Atherosclerosis risk factors: the possible role of homocysteine. Mol Cell Biochem 2000; 207: 143-8.

30. Ruiz-Fernández N, Espinoza M, Barrios E, Reigosa A. Factores Cardiometabólicos en una Comunidad de Valencia, Venezuela. Rev Salud Pública 2009; 11: 383-94

31. Vermaak W, Ubbink J, Barnard H, Potgierter G, Jaarsveld H, Groenewald A. Vitamin B6 nutrition status and cigarette smoking. Am J Clin Nutr 1990; 51: $1058-61$.

32. Koudstaal P, Grobbee D. Homocysteine and Short-term risk of myocardial infarction and stroke in the elderly. The Rotterdam study. Arch Intern Med 1999; 159: 38-43

33. Ades PA, Savage PD, Harvey-Berino J. The Treatment of Obesity in Cardiac Rehabilitation. J Cardiopulm Rehabil 2010; 30: 289-98.

34. Barja S, Arteaga A, Acosta A, Hodgson M. Resistencia insulínica y otras expresiones del síndrome metabólico en niños obesos chilenos. Rev Méd Chile 2003; 131: 259-68.

35. Organización Panamericana de la Salud/Organización Mundial de la Salud Evaluación económica de políticas públicas para el control del tabaquismo en Venezuela. Caracas: OPS/OMS; 2004.

36. Brandi P. Diabetes mellitus and dislipidemias. Can J Diabetes Care 1998; 22: 28-38.

37. Libby P, Ridker PM, Maseri A. Inflammation and atherosclerosis. Circulation 2002; 105: 1135-43. 
38. Gotthelf S, Jubany L. Prevalencia de factores de riesgo asociados al síndrome metabólico en niños y adolescentes obesos de la ciudad de Salta. Centro Nacional de Investigaciones Nutricionales. Salta, Argentina. Foro Latinoamericano de Nutrición [Internet] 2004 [citado el 6 de junio de 2011] Pp. 1-16. Disponible en: http://latinut.net/documentos/obesidad/docuybase/SM\%20trabajo\%20publicado. PDF. 2004.

39. Chamberlain A, Agarwal S, Ambrose M, Folsom A, Soliman E, Alonso A. Metabolic syndrome and incidence of atrial fibrillation among blacks and whitesin the Atherosclerosis Risk in Communities (ARIC) Study. Am Heart J 2010; 159: 850-6.

40. Bonakdaran S, Ebrahimzadeh S, Noghabi S. Cardiovascular disease and risk factors in patients with type 2 diabetes mellitus in Mashhad, Islamic Republic of Iran. East Mediterr Health J 2011; 17(9): 640-6.

41. Janszky I, Vatten L, Romundstad P, Laugsand L, Bjørngård J, Mańczuk M, et al. Metabolic syndrome in Poland - the PONS Study. Ann Agric Environ Med 2011; 18(2): 270-2.

42. Lee J, Bae J, Park J, Park C, Youn H, Choi D, Ahn Y, et al. Morning hypertension in treated hypertensives: baseline characteristics and clinical implications. Korean Circ J 2011; 41(12): 733-43.

43. Oh S. Obesity and metabolic syndrome in Korea. Diabetes Metab J 2011; 35(6): 561-6.
44. Becerra L, Lenin V,Arata-Bellabarba G, Velázquez-Maldonado E. Prevalencia del síndrome metabólico en la población urbana de mucuchíes, Mérida Venezuela. Rev Venez Endocrinol Metab 2009; 7: 16-22.

45. Bencomo M, Velázquez-Maldonado E, Arata-Bellabarba G, Villarroel V. Componentes del síndrome plurimetabólico en tres poblaciones de Los Andes Venezolanos. Rev ALAD 2002; 10: 85-93.

46. Luengo E, Ordóñez B, Bergua C, Laclaustra M. Síndrome metabólico: retos y esperanzas. Obesidad, dislipemia y síndrome metabólico. Rev Esp Cardiol Supl 2005; 5: 21D-9D.

47. Martínez O, Castrillón V. Diabetes mellitus, factor de riesgo de severidad de enfermedad isquémica crítica aterosclerótica y de viabilidad de miembros inferiores. Acta Med Colomb 2010; 35: 39-46.

48. Consenso Nacional de Diabetes tipo 2. Sociedad Venezolana de Endocrinología y Metabolismo. 2003

49. Choi S, Ahn C, Cha B, Chung Y, Lee K, Lee H, et al. The prevalence of the metabolic syndrome in Korean adults: comparison of WHO and NECP criteria. Yonsei Med J 2005; 30: 198-205.

50. McConnell T, Santamore W, Larson S, Homko C. Rural and Urban Characteristics Impact Cardiovascular Risk Reduction. Cardiopulm Rehabil Prev 2010; 30: 373. 